\title{
Routing Techniques in Wireless Sensor Networks
}

\author{
Sanjolly Jain \\ Research Scholar \\ SBSSTC, Ferozepur, Punjab
}

\author{
Amit Grover \\ Assistant Professor \\ SBSSTC, Ferozepur, Punjab
}

\begin{abstract}
Wireless sensor network is a self configured network being composed of a large number of sensors. Due to the fact that sensors in the wireless sensor network are powered with battery and it is difficult to replace and/or recharge their batteries, energy efficient routing is the major concern in the field of wireless sensor network to enhance the lifetime of the network. Consequently, Numbers of routing techniques have been proposed for wireless sensor network to make longer life time and low energy consumption. Mainly these are sorted into three categories such as Flat and data centric routing, Hierarchical routing, Location based routing. This paper presents a review of some major work in area of flat and data centric routing technique and hierarchical routing technique for WSNs. This article also compares the characteristics and performance issues of different routing protocols.
\end{abstract}

\section{General Terms}

Routing Techniques in Wireless Sensor Networks

\section{Keywords}

Wireless Sensor Network, Flat Routing, Data-centric Routing, Hierarchical Routing Techniques

\section{INTRODUCTION}

With the rapid development of micro-electromechanical systems and the wireless communication, have reduced the cost of the deployment of small sensor nodes. WSN is a network of many tiny, low power devices called nodes, which provide opportunities in several domains ranging from military to civil use [1] [2]. Considering the fact that the longevity of wireless sensor network is usually limited due to their non-rechargeable energy resources, thus it need be carefully planned to enhance the network lifetime. The main source of power consumption is communication [3], hence a high performance routing technique is an important part in WSN. According to the structure of network topology, routing techniques are divided into three categories such as datacentric and flat architecture routing technique, hierarchical routing technique and location based routing technique. This survey paper discusses the data-centric and flat architecture routing technique and the hierarchical routing technique along with routing protocols that follow the mechanism of these routing techniques. This paper also provides the comparison of various routing protocols in terms of various factors in a tabular form.

\section{DATA-CENTRIC AND FLAT ARCHITECTURE ROUTING TECHNIQUE}

A WSN network consists of a large number of sensor nodes, so it is very difficult to provide specific Ids to each of the sensor nodes. This is the main reason addressed based routing protocols are not desirable for WSNs. To vanquish this problem, data centric and flat architecture routing protocols have been developed. In data centric routing, sink initiates the query message to the destination region and then waits for the data from the sensor nodes deployed in that region. Instead of sensor nodes Ids, data centric routing requires attribute based naming [4]. Figure 1 shows the data centric routing. In this case, sink is interested in regions where the temperature is greater than $70 \mathrm{~F}$. Hence, the nodes with sensor readings matches this query are reported. Kindly note that data centric routing assigns routes on the basis of the query content and hence for each query, the nodes that provide information will change. In flat architecture routing, there are a large number of nodes that are integrated together to sense the environment. These nodes are similar to each other and there is no need to assign global Ids to them. Note that every node within the selected region is busy in the routing mechanism hence these nodes can apply any routing rule to the packets that is either forward or drop [5].

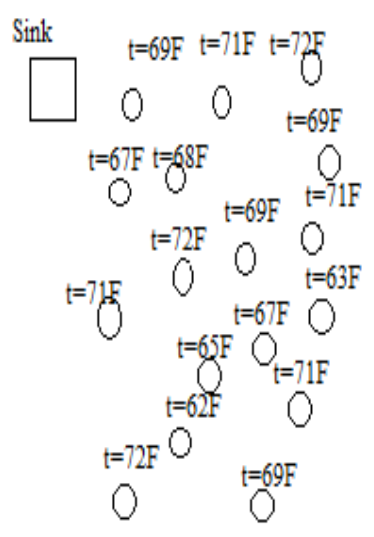

(a) WSN topology with temperature reading of each node

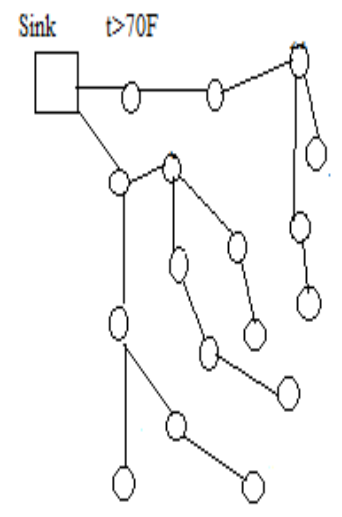

(b) Sink initiates a Query (t>70F) 


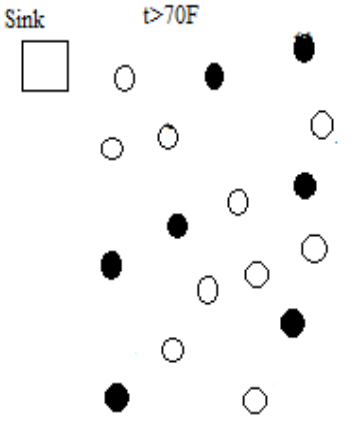

(c) Nodes with matching readings are addressed (d) Routes are generated

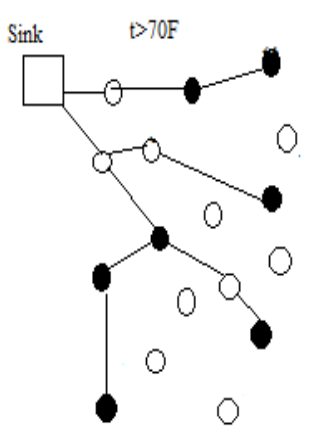

Figure 1: Illustration of data-centric routing

In the next subsection, paper discusses the protocols that may follow flat and data centric routing principles and also highlights their advantages and their performance issues.

\subsection{Flooding}

Flooding is the simplest routing technique which has been designed for multi-hop networks. In flooding, whenever a node receives a packet, it sends out the received packet to all of its neighbors. This process continues until all the nodes within the network receive the packet or a maximum number of hops for that packet are achieved [6]. In other words we can say that in flooding, the received packets are flooded through the whole network. The main advantage of flooding is that all the nodes that are participating in this routing technique don't have a need for neighborhood information. However this routing technique has many flaws as explained below:

\subsubsection{Implosion}

Is the process in which multiple nodes send out same packet to the same destination. Due to this fact, duplicated packets are received by the destination node. As shown in figure if node $\mathrm{X}$ shares $\mathrm{M}$ neighbor nodes with another node $\mathrm{Y}$ the sensor node $\mathrm{Y}$ receives $\mathrm{M}$ copies of the packets sent by node $\mathrm{X}$ as shown in figure 2(a).

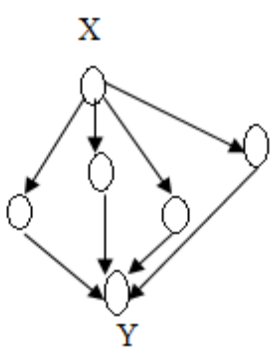

(a) Implosion

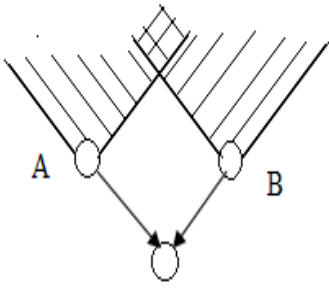

$\mathrm{C}$

(b) Data Overlap
Figure 2: Main problems with flooding

\subsubsection{Overlap}

Sometimes the two sensing nodes deals with overlapping sensing networks therefore both the nodes sense the same data at the same time. Consequently their neighbour nodes will get replicated data as shown in the figure 2(b).

\subsubsection{Resource blindness}

Availability of energy is the most dominant resource in WSNs which should be significantly utilized by the networking protocols. However, the flooding nodes do not deal with the amount of energy resources provides to them at the given time of interval.

\subsection{Gossiping}

Gossiping protocol was proposed to overcome the implosion problem faced by flooding nodes. In gossiping, whenever a node receives data, it doesn't broadcast the data through the whole network but select a random node among its neighbors and then send the data to that randomly selected node. Once the selected neighbor node receives the data then it again selects a random node among its neighbor and sends the data to that node. Note that, at a particular time interval only a single node is informed about the data. Hence, the information is distributed slowly and long time propagation is required to forward the data to all sensor nodes [7]. On the other hand, the energy consumption of gossiping protocol is less than that of flooding as it avoids the replicated copies of packet. Although, gossiping can avoid the implosion problem but it cannot solve the overlapping problem.

\subsection{Direct Diffusion}

It can be classified under both data centric and flat routing protocols. A node appeals for data by forwarding interests for naming data. A sensing work is propagated within the whole network. The nodes which show interest, arranged their own gradients throughout the network to which the data transmission is carried out [8]. During the delivery of data, reinforcement and negative reinforcement techniques are used for systematic distribution. As shown in the figure 3 .
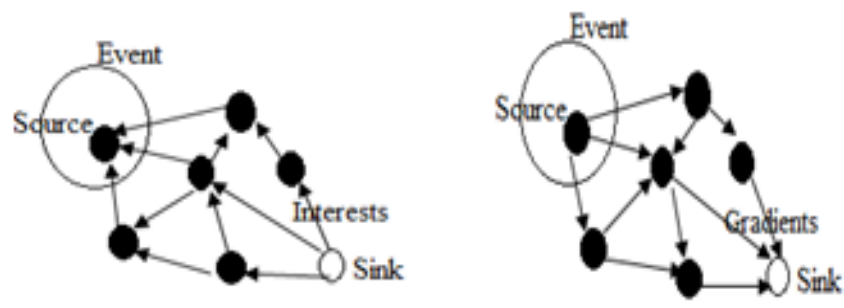

(b)

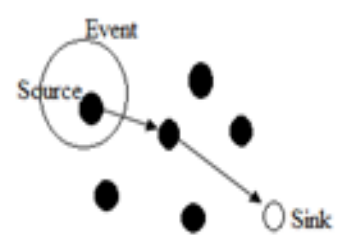

(c)

Figure 3: Direct dffusion 
SPIN[9], energy aware routing protocols[10], rumor routing[11], gradient-based Routing[12], CADR[13], COUGAR[14], ACQUIRE[15], Short path minded SPIN(SPMS)[16] and solar aware routing[17] also follow flat and data centric routing principles

\section{HIERARCHICAL ROUTING TECHNIQUE}

In hierarchical routing, the whole network is divided into multiple clusters. One node in each cluster plays a leading role called cluster-head. The cluster-head is the only node that can communicate with a base station in clusters routing protocols [18]. This significantly reduces the routing overhead of normal nodes because normal nodes have to transmit to cluster-head only. In the next subsection, paper summarizes the protocols that may follow hierarchical routing principles and also highlights their advantages and their performance issues.

\subsection{LEACH (Low Energy Adaptive Clustering Hierarchy) Routing Protocol} LEACH (Low Energy Adaptive Clustering Hierarchy) refines the energy consumption in WSNs through cluster based procedure. LEACH selects sensor node randomly as cluster head and creates clusters in the network then, every non-CH sensor joins the cluster of closest $\mathrm{CH}$. The transmission within the clusters is directed to the cluster heads, which accomplish aggregation, then communicate with sink. Consequently $\mathrm{CHs}$ consume more energy than non-CHs, due to this fact LEACH permits rotation of $\mathrm{CH}$ status to balance the energy consumption among sensors [19]. The working of LEACH is managed through the rounds. At every round, the operation of LEACH is divided into two stages, the set up stage and the steady state stage. In set up time, $\mathrm{CHs}$ are elected, clusters are created and then cluster communication schedule is determined. Firstly, sensor nodes choose a random number $\mathrm{m}$ between 0 and 1 . If this number $m$ is less than threshold value $\mathrm{T}(\mathrm{n})$, then sensor nodes become the cluster head. Since the number $\mathrm{m}$ is randomly selected, that's why the number of CHs cannot be fixed. T (n) is calculated as

$$
T(n)= \begin{cases}\frac{p}{1-p\left(\operatorname{rmod} \frac{1}{p}\right)} & , n \in G \\ 0 & , \text { otherwise }\end{cases}
$$

Where $\mathrm{p}$ is the desired percentage of $\mathrm{CHs}, \mathrm{r}$ is the present round and $\mathrm{G}$ is the set of nodes that haven't been $\mathrm{CHs}$ in the last $1 / p$ rounds. The elected $\mathrm{CHs}$ then broadcast the advertisement message to their neighbors within the network, to inform that they are the latest $\mathrm{CHs}$. For this procedure, the CSMA random access technique is used to avoid the collisions. Once the sensor nodes receive the advertisement message, then they decide the cluster they want to join. If a sensor node earns multiple advertisement messages from multiple $\mathrm{CHs}$, then the selection of cluster head is done on the basis of the signal strength of the advertisement message. The cluster head with the highest signal strength is elected. After this, the sensor nodes inform the associate $\mathrm{CH}$ that they will be the member of the cluster. This is called the cluster setup phase. Ultimately, the schedule creation operation is performed, where the $\mathrm{CHs}$ allocate the time during which the sensor nodes can send data to the CHs. A time division multiple access technique is used for this selection. After the completion of the setup phase, LEACH switches to steady state phase. In this phase, the sensor nodes start sensing and transmitting the data to the $\mathrm{CHs}$. $\mathrm{CH}$ s aggregate the data from the sensor nodes within their cluster then finally send all the data to the sink. However, this routing technique has also some limitations as explained below

- The procedure for the selection of cluster heads doesn't use energy. Consequently, low power nodes can become $\mathrm{CHs}$ and these nodes die faster and produce a disconnection between the sink and WSN.

- LEACH is not applicable to those WSNs that are deployed in large regions.

- At every round, the number of $\mathrm{CHs}$ formation is unpredictable due to the randomly selected value $\mathrm{m}$.

All these disadvantages are overcome by descendents of LEACH protocols. Some of these are LEACH-C [20], LEACH-F [21], LEACH-B [22], LEACH-E [23], A-LEACH [24], S-LEACH [25] and LEACH-H [26].

\subsection{PEGASIS (Power Efficient Gathering in Sensor Information System) Routing Protocol}

PEGASIS (Power Efficient Gathering in Sensor Information System) is a chain based power efficient protocol [27]. The construction of chain is done according to a greedy algorithm, where each node selects their closest neighbor as the next hop in the chain. The main features of PEGASIS are:

- It assumes that all the nodes have global knowledge about the network.

- Chain construction starts from the nodes that are farthest from the sink.

Communication within the chain is carried out sequentially. Each node within the chain collects data from its neighbor until all the data are gathered at one sensor node called chain leader. Chain leader manages the communication order by passing the token among the nodes [28]. The chain communication of PEGASIS is shown in figure 4. 


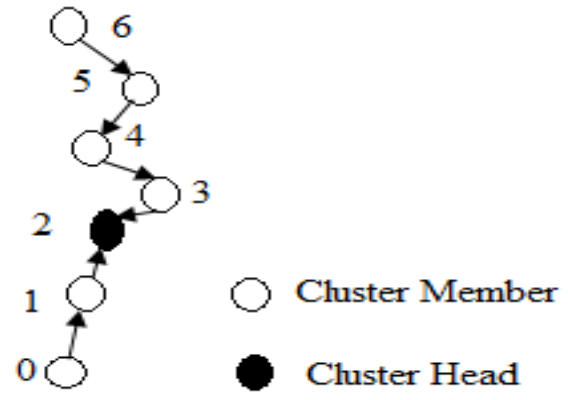

Figure 4: Chain structure of PEGASIS

Here, node 2 is the chain leader. Firstly, node 2 passes the token to node 0 to initiate the communication. Node 0 sends out its data to node 1 , which fuses this data along with its own to form a packet. This packet is then transmitted to the node 2 . Once the node 2 receives the packet from node 1, it passes the token to the other end of the chain i.e., node 6. Data from sensor nodes 6, 5, 4 and 3 is also collected and transmitted to node 2 using the same procedure. After receiving all the information, node 2 transmits the data to sink through a single hop communication.

\subsection{TEEN and APTEEN Hierarchical} Routing Protocol

LEACH and PEGASIS protocol support applications where information from sensor nodes is periodically communicated to the sink. Consequently, these protocols may not be approachable to event based applications, where information is communicated only when certain events occur. TEEN (Threshold Sensitive Energy Efficient Sensor Network) protocol was proposed [29] to overcome this problem, the aim of TEEN protocol is to support an event based delivery in the network. TEEN organized the nodes into multiple levels of hierarchy. It follows a hierarchical model along with datacentric algorithms. At first, the clusters are formed then; each $\mathrm{CH}$ transmits two threshold values to all the nodes. These are hard and soft threshold for the sensed attributes. The hard threshold is the least possible value of an attribute depends on the sensor will be communicating data to the sink. When the sensed value of attribute is greater than the threshold, the data are transmitted to the cluster-head. This allows the nodes to transmit only relevant data. Once a value above the hard threshold is sensed, then the node confirms, if the difference in the latest and earlier value is greater than the soft threshold; if so the new data are communicated.

Since TEEN is depending on fixed threshold limits, it is not suitable for periodic reports required by some applications. To provide periodic information, APTEEN (Adaptive ThresholdSensitive Energy Efficient Sensor Network) protocol has been developed. APTEEN provides a TDMA- based structure for data transmission in every cluster. As a result, each node sends its data periodically to the cluster-head. Both, event based and monitoring applications can be served by APTEEN [30].

\section{QUALITATIVE EVALUATION OF ROUTING PROTOCOLS}

Data centric and flat architecture routing mechanisms suffer from data overload close to sink as the majority of information being concentrated near the sink. As a result, these nodes consume more energy than other sensor nodes hence, die faster and create disconnection in WSN. The limitations of data-centric and flat architecture routing protocols can be addressed by forming a hierarchical routing technique where nodes are grouped into clusters and the interactions between the cluster members are controlled by cluster-head. Based on this mechanism, many hierarchical routing protocols have been proposed to address the scalability and energy consumption challenges of WSN. Table 1 shows the comparison of different routing protocols in WSN according to different metrics. 
Table 1. Comparison of different routing protocols in WSN

\begin{tabular}{|c|c|c|c|c|c|c|c|c|}
\hline $\begin{array}{c}\text { Routing } \\
\text { Protocols }\end{array}$ & Classification & $\begin{array}{c}\text { Position } \\
\text { awareness }\end{array}$ & Scalability & Mobility & $\begin{array}{c}\text { Power } \\
\text { usage }\end{array}$ & Aggregation & $\begin{array}{c}\text { Query } \\
\text { based }\end{array}$ & $\begin{array}{c}\text { QoS } \\
\text { Flooding }\end{array}$ \\
Flat & No & Limited & No & High & No & No & No \\
\hline Gossiping & Flat & No & Limited & No & High & No & No & No \\
\hline $\begin{array}{c}\text { Direct } \\
\text { Diffusion }\end{array}$ & $\begin{array}{c}\text { Data centric/ } \\
\text { flat }\end{array}$ & No & Limited & Limited & Limited & Yes & Yes & No \\
\hline LEACH & Hierarchical & No & Good & Fixed BS & Maximum & Yes & No & No \\
\hline PEGASIS & Hierarchical & No & Good & Fixed BS & Maximum & No & No & No \\
\hline $\begin{array}{c}\text { TEEN \& } \\
\text { APTEEN }\end{array}$ & Hierarchical & No & Good & Fixed BS & Maximum & Yes & No & No \\
\hline
\end{tabular}

\section{CONCLUSION}

In recent years, routing in wireless sensor network has become modern area of research. One of the main problems in the design of routing protocols for WSNs is how to efficiently utilize energy because energy resources are very limited. The main aim behind the routing protocols design is to keep the sensors in operation as long as possible, thus extending the network's lifetime. This paper, presented a survey of routing techniques in wireless sensor networks. These have the common objective of trying to extend the lifetime of the sensor network. Although many routing protocols have been proposed in WSN, more work is still needed to find more efficient, scalable and robust routing scheme to reduce energy consumption and prolonging network's lifetime in small and large WSNs.

\section{REFERENCES}

[1] J. Yick, B. Mukherjee, and D. Ghosal, Wireless sensor network survey, Computer Networks, Vol. 52, No. 12, 2292-2330, 2008.

[2] L. Sun, J. Li, Y. Chen, et al., Wireless Sensor Network, Tsinghua University Press, Beijing, China, 2005.

[3] D. Estrin, Tutorial "Wireless Sensor Networks" Part IV:Sensor Network Protocols, Mobicom, 23-28, 2002.

[4] J. Mirkovie, G.P. Venkataramani , S. Lu and L. Zhang," A Self Organized Approach to Data Forwarding In Large Scale Sensor Network," In Proceedings of IEEE International Conference on Communications (ICC'01), Helsinki, Finland, June 2001.

[5] C. Shen, C. Srisathapornphat and C. Jaikaeo," Sensor Information Networking Architecture and Applications," IEEE Personal Communications , 8(4): 52-59, August 2001.

[6] W. R. Heinzelman, J. Kulik, and H. Balakrishnan, "Adaptive Protocols for Information Dissemination in Wireless Sensor Networks," in proc. ACM Mobi Com '99, Seattle, WA, 1999.
[7] S. M. Hedetniemi, S. H. Hedetniemi, and A. Liestman,"A Survey of Gossiping and Broadcasting in Communication Networks", Networks, vol. 18, 1988.

[8] C. Intanagonwiwat, R. Govindan, D. Estrin, J. Heidemann, and F. Silva, "Directed Diffusion for Wireless Sensor Networking", IEEE/ACM Transactions on Networking, vol.11, pp. 2-16, Feb. 2003.

[9] J. Kulik, W. R. Heinzelman, and H. Balakrishnan, "Negotiation-based protocols for disseminating information in wireless sensor networks", Wireless Networks, Volume: 8, pp. 169-185, 2002.

[10] J. Aslam, Q. Li, and D. Rus," Three Power Aware Routing Algorithms for Sensor Networks", Wireless Communications and Mobile Computing, 3(2): 187-208, 2003.

[11] D. Braginsky and D. Estrin," Rumor Routing Algorithm for Sensor Networks", in the Proceedings of the First Workshop on Sensor Networks and Applications (WSNA), Atlanta, GA, October 2002.

[12] C. Schurgers and M.B. Srivastava, "Energy efficient routing in wireless sensor networks", in the MILCOM Proceedings on Communications for Network-Centric Operations: Creating the Information Force, McLean, VA, 2001.

[13] M. Chu, H. Haussecker, and F. Zhao," Scalable Information-Driven Sensor Querying and Routing for ad hoc Heterogeneous Sensor Networks", The International Journal of High Performance Computing Applications, Vol. 16, No. 3, August 2002.

[14] Y. Yao and J. Gehrke, "The cougar approach to innetwork query processing in sensor networks", in SIGMOD Record, September 2002.

[15] N. Sadagopan et al., The ACQUIRE mechanism for efficient querying in sensor networks, in the Proceedings of the First International Workshop on Sensor Network 
Protocol and Applications, Anchorage, Alaska, May 2003.

[16] G. Khana, S. Bagchi and Y. Wu. Fault Tolerant," Energy Aware Data Dissemination Protocol In Sensor Network", In Proceedings of the International Conference On Dependable System And Networks, pp. 795-804, Florence, Italy, June 2004.

[17] T. Voigt, H. Ritter and J. Schiller, "Solar Aware Routing In Wireless Sensor Networks", In Proceedings of Personal Wireless Communication 2003, Venice, Italy, September 2003.

[18] S. K. Singh, M. P. Singh, and D. K. Singh ,"A Survey of Energy- Efficient Hierarchical Cluster-based Routing in Wireless Sensor Networks, "International Journal of Advanced Networking and Application (IJANA), vol. 02,issue 02,pp 570-580,Sept 2010.

[19] W. Heinzelman, A. Chandrakasan, and H. Balakrishnan, "Energy-Efficient Communication Protocol for Wireless Micro-Sensor Networks", in Proc. of the 33rd Annual Hawaii International Conf. on System Sciences, pp. 3005-3014, 2000.

[20] W. Heinzelman, A. Chandrakasan, and H. Balakrishnan, "An application-specific protocol architecture for wireless microsensor networks," IEEE Transaction on Wireless Communications, vol. 1, no. 4, pp. 660670,2002 .

[21] W. B. Heinzelman, "Application- Specific Protocol Architectures for Wireless Networks" PhD thesis, Massachusetts Institute of Technology, June 2000.

[22] Depedri, A. Zanella and R. Verdone, "An Energy Efficient Protocol for Wireless Sensor Networks" In Proc. AINS, pp. 1-6, 2003.

[23] X. Fan and Y. Song, "Improvement on leach protocol of wireless sensor network," in In Proceedings of the International Conference on Sensor Technologies and Applications, pp. 260-264,2007.

[24] M.S. Ali, T. Dey and R. Biswas "ALEACH: Advanced LEACH Routing Protocol for Wireless Microsensor Networks" International Conference on Electrical and Computer Engineering, pp. 909-914.

[25] W. Xiao-yun, Y. Li-zhen1, and C. Ke-fei1, "SLEACH: Secure low-energy adaptive clustering hierarchy protocol for wireless sensor networks," vol. 10, pp. 127-131, 2005.

[26] W. Wang, Q. Wang, W. Luo, M. Sheng, W. Wu and Li Hao "Leach-H: An Improved Routing Protocol for Collaborative Sensing Networks" International
Conference on Wireless Communications \& Signal Processing, pp.1-5, 2009.

[27] S. Lindsey, C. S. Raghavendra,"PEGASIS: PowerEfficient Gathering in Sensor Information Systems," presented at Proc. of IEEE Aerospace Conference, Montana, 2002

[28] S. Lindsey, C.S. Raghavendra and K. Sivalingam," Data Gathering In Sensor Networks Using The Energy* Delay Metric", In proceeding of the IPDPS workshop on Issues in Wireless Networks and Mobile Computing, San Francisco, USA, April 2001.

[29] Manjeshwar and D. P. Agarwal, "TEEN: a routing protocol for enhanced e \pm ciency in wireless sensor networks," In1st International Workshop on Parallel and Distributed Computing Issues in Wireless Networks and Mobile Computing, April 2001.

[30] Manjeshwar and D. P. Agarwal, "APTEEN: A hybrid protocol for e \pm cient routing and comprehensive information retrieval in wireless sensor networks," Parallel and Distributed Processing Symposium., Proceedings International, IPDPS, pp. 195-202, 2002.

\section{AUTHOR'S BIOGRAPHY}

Sanjolly Jain received her B. Tech degree in Electronics and Communication Engineering in 2012 from North West Institute of Engineering and Technology, Affiliated to Punjab Technical University, Kapurthala, Punjab, India. Her place of birth is Ferozepur, Punjab, India on 22nd December 1990. At present she has been doing her research work under the guidance of Mr. Amit Grover, Assistant Professor, Department of ECE, Shaheed Bhagat Singh State Technical Campus, Moga road, Ferozepur-152004, Punjab, India.

Amit Grover (M'06-SM'09-PI'11\&12) The author became a Member (M) of Association ISTE in 2006, a Senior Member (SM) of society SELCOME in September 2009, and a ProjectIn charge (PI) in august 2011 and in September 2012. The author place of birth is Ferozepur, Punjab, India on 27th, September 1980.The author received his M. Tech degree in Electronics and Communication Engineering from Punjab Technical University, Kapurthla, Punjab, India in 2008 and received his B. Tech degree in Electronics and Communication Engineering from Punjab Technical University, Kapurthala, Punjab, India in 2001. Currently, he is working as an Assistant Professor in Shaheed Bhagat Singh State Technical Campus, Ferozepur, Punjab, India. The author is a Reviewer of many International Journals. His area of interest includes signal processing, MIMO systems, Wireless mobile communication, High speed digital communications, 4G Wireless Communication and VLSI Design. 